\title{
AN APPLICATION OF STEINBERG'S CONSTRUCTION OF TWISTED GROUPS
}

\section{Eivind Stensholt}

\begin{abstract}
The construction of the twisted groups uses automorphisms of certain Chevalley groups derived from symmetries of the Dynkin diagrams. This paper applies the same method to the symmetries of the extended Dynkin diagrams. The groups so constructed turn out to be other Chevalley groups exhibited in a way which let a particular subgroup structure appear.
\end{abstract}

Introduction. Let $\Phi$ be an $n$-dimensional indecomposable crystallographic root system of one rootlength. Steinberg's construction of the twisted Chevalley groups makes use of a nontrivial isometry of $\Phi$ which permutes the roots of a given fundamental system. Such isometries exist except for $\Phi=A_{1} ; E_{7} ; E_{8}$ and are defined by symmetries of the Dynkin diagrams.

This paper applies the same method to another isometry of $\Phi$ which permutes the roots of a set $S=\left\{r_{1}, \cdots, r_{n}, M\right\}$ of $n+1$ roots where $\left\{r_{1}, \cdots, r_{n}\right\}$ is a fundamental system and $S$ has the following properties in common with a fundamental system:

$$
\left(M, r_{i}\right) \leqq 0, \quad 1 \leqq i \leqq n .
$$

(0.2) The graph with the $n+1$ roots of $S$ as nodes, $r$ and $s$ being connected if and only if $(r, s)<0$, is a tree.

The condition (0.1) shows that $-M$ is in the fundamental chamber defined by $\left\{r_{1}, \cdots, r_{n}\right\}$, and because all roots in $\Phi$ are conjugates, $M$ is uniquely determined as the lowest root. Therefore the graph is the extended Dynkin diagram as defined by Bourbaki [2, p. 198]. The cases that satisfy $(0.2)$ are $\Phi=D_{n} ; E_{7} ; E_{6}$. The extended diagrams are:
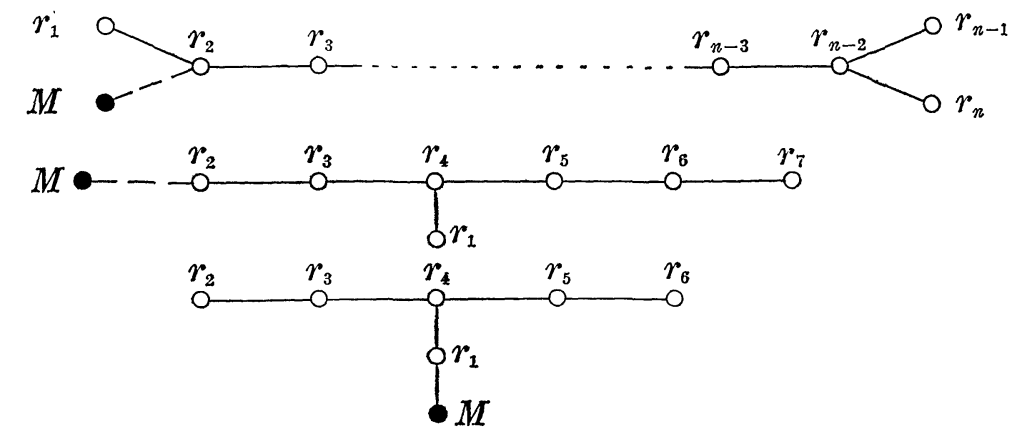

The extended diagrams suggest the definition of an isometry $\eta$ 
of $\Phi$. The following five special cases will be treated: $\Phi=D_{m+2} ; D_{2 m}$; $D_{2 m+1} ; E_{7} ; E_{6}(m \geqq 2)$ and $\eta=\lambda ; \mu ; \nu ; \varphi ; \psi$ respectively, where

$$
\begin{aligned}
\lambda: & r_{i} \longrightarrow r_{i} \text { for } 2 \leqq i \leqq n-2, r_{n-1} \longleftrightarrow r_{n}, r_{1} \longrightarrow M \\
& (n=m+2) \\
\mu, \nu: & r_{i} \longrightarrow r_{n-i} \text { for } 1 \leqq i \leqq n-1, r_{n} \longrightarrow M \\
\varphi: & r_{1} \longrightarrow r_{1}, r_{4} \longrightarrow r_{4}, r_{3} \longleftrightarrow r_{5}, r_{2} \longleftrightarrow r_{6}, r_{7} \longrightarrow M \\
\psi: & r_{4} \longrightarrow r_{4}, r_{1} \longrightarrow r_{3} \longrightarrow r_{5} \longrightarrow r_{1}, r_{2} \longrightarrow r_{6} \longrightarrow M .
\end{aligned}
$$

Because the root $M$ is uniquely determined by $\left\{r_{1}, \cdots, r_{n}\right\}, \lambda(M)=r_{1}$, $\mu(M)=r_{n}, \nu(M)=r_{n}, \varphi(M)=r_{7}, \psi(M)=r_{2}$. Hence $\eta$ is an isometry of order

$$
|\eta|=2 ; 2 ; 2 ; 2 ; 3 \text { for } \eta=\lambda ; \mu ; \nu ; \varphi ; \psi \text { respectively. }
$$

About certain automorphisms of Chevalley groups we state a few facts that are easily checked:

Let $G_{1}$ and $G_{2}$ be Chevalley groups defined by the same indecomposable root system and the same finite field $G F(q), G_{1}$ universal, and let $\gamma$ be a homomorphism of $G_{1}$ onto $G_{2}$ with kernel in $Z\left(G_{1}\right)$. Further let $\alpha_{1} \in$ Aut $G_{1}$ and $\alpha_{2} \in$ Aut $G_{2}$ be either (a) field automorphisms or (b) products of field and graph automorphisms as used in Steinberg's twisting construction, and such that $\alpha_{2} \gamma=\gamma \alpha_{1}$.

Let $\widetilde{G}_{i}$ be the fixpointgroup of $\alpha_{i}$ in $G_{i}, i=1,2$. Then $\widetilde{G}_{2}$ is generated by $\gamma\left(\widetilde{G}_{1}\right)$ and $\alpha_{2}$-invariant elements of the diagonal subgroup in $G_{2}, \gamma\left(\widetilde{G}_{1}\right) \triangleleft \widetilde{G}_{2}$ and the index $\left[\widetilde{G}_{2}: \gamma\left(\widetilde{G}_{1}\right)\right]$ is prime to $q$. The inclusion may be proper.

If $G_{2}$ is adjoint, $\gamma\left(\widetilde{G}_{1}\right)$ is in case (b) a twisted group which is simple with a few exceptions. If $G_{2}$ is not adjoint, $\gamma\left(\widetilde{G}_{1}\right)$ is in general a central extension of the mentioned twisted group. We will still call it a twisted group and denote it by the usual symbol, ${ }^{2} A_{n}\left(q^{2}\right)$ etc.

The content of the paper is as follows.

Section 1 describes the $\eta$-orbits of the roots in $\Phi$. Let $\operatorname{Pr}$ be the orthogonal projection of the vectorspace spanned by $\Phi$ onto the subspace of $\eta$-invariant points. $\operatorname{Pr}^{-1}(0)$ contains a root system $\Phi^{\mathrm{ker}}$. It is shown that $\operatorname{Pr}(\Phi)-\{0\}$ is a root system, and it will be denoted by $\Phi^{P r}$. Subsystems $\Phi_{i}^{\text {sub }}, i=1, \cdots, N$, that are conjugates under $W\left(\Phi^{\mathrm{ker}}\right)$, the reflection group defined by $\Phi^{\mathrm{ker}}$, are defined such that

$$
\eta\left(\Phi_{i}^{\text {sub }}\right)=\Phi_{i}^{\text {sub }}, \quad \Phi_{i}^{\text {sub }} \cap \Phi^{\mathrm{ker}}=\varnothing, \quad \operatorname{Pr}\left(\Phi_{i}^{\text {sub }}\right)=\Phi^{P r} .
$$

Let $\mathscr{L}(\Phi)$ be the simple complex Lie algebra defined by $\Phi$, and let $\mathscr{L}\left(\Phi_{i}^{\text {sub }}\right), \mathscr{L}\left(\Phi^{\text {ker }}\right)$ be the subalgebras supported by $\Phi_{i}^{\text {sub }}, 1 \leqq i \leqq N$, 
and $\Phi^{\text {ker }}$. Similarly, let $\Phi(q)$ be a Chevalley group defined by $\Phi$ and the field $G F(q)$, and let $\Phi_{i}^{\text {sub }}(q)$, $\Phi^{\mathrm{ker}}(q)$ be subgroups. In $\S 2$ automorphisms of $\mathscr{L}(\Phi)$ and $\Phi\left(q^{|\eta|}\right)$ corresponding to $\eta$ are defined. They are shown to be of order as in (0.5) and will also be denoted by $\eta=\lambda ; \mu ; \nu ; \varphi ; \psi$. The subalgebras and subgroups introduced above are $\eta$-invariant, and the group automorphism $\eta$ is so defined that the restrictions $\eta \mid \Phi_{i}^{\text {sub }}\left(q^{|\eta|}\right)$ are the usual twisting automorphisms, defining subgroups of the types

$$
\begin{gathered}
{ }^{2} D_{m+1}\left(q^{2}\right) ;{ }^{2} A_{2 m-1}\left(q^{2}\right) ;{ }^{2} A_{2 m}\left(q^{2}\right) ;{ }^{2} E_{6}\left(q^{2}\right) ;{ }^{3} D_{4}\left(q^{3}\right) \\
\quad \text { for } \eta=\lambda ; \mu ; \nu ; \varphi ; \psi \text { respectively. }
\end{gathered}
$$

Then $\S \S 3$ and 4 establish the following main result:

(0.8) THEOREM. The $\Phi\left(q^{\left|\eta_{\mid}\right|}\right)$-automorphism $\eta$ is conjugate in Aut $\Phi\left(q^{|\eta|}\right)$ to a field automorphism if $\eta \neq \nu$ and to the product of a graph-and a field automorphism if $\eta=\nu$.

Hence the fixpointgroup of $\eta$ is or contains (see the discussion above) a group of one of the types

$$
\begin{aligned}
& D_{m+2}(q) ; D_{2 m}(q) ;{ }^{2} D_{2 m+1}\left(q^{2}\right) ; E_{7}(q) ; E_{6}(q) \\
& \quad \text { for } \eta=\lambda ; \mu ; \nu ; \varphi ; \psi \text { respectively. }
\end{aligned}
$$

As a consequence of $(0.9)$ and (0.7) one obtains the embeddings

$$
\begin{array}{cl}
{ }^{2} D_{m+1}\left(q^{2}\right) \subset D_{m+2}(q) \\
{ }^{2} A_{2 m-1}\left(q^{2}\right) \subset D_{2 m}(q), & { }^{2} A_{2 m}\left(q^{2}\right) \subset{ }^{2} D_{2 m+1}\left(q^{2}\right) \\
{ }^{2} E_{6}\left(q^{2}\right) \subset E_{7}(q), & { }^{3} D_{4}\left(q^{3}\right) \subset E_{6}(q) .
\end{array}
$$

In $\$ 5$ these embeddings are described somewhat closer. The paper aims at a unified presentation of the arguments, with special treatment of the individual cases $\eta=\lambda ; \mu ; \nu ; \varphi ; \psi$ only when technical reasons call for it.

1. The isometry of the root system. We first derive some information about the isometry $\eta$ of $\Phi$ defined in (0.4). This information is given in the following table, using the notation of the introduction.

\begin{tabular}{llclll}
$\eta$ & \multicolumn{1}{c}{$\Phi$} & $\operatorname{dim} \operatorname{Pr}^{-1}(0)$ & $\Phi^{\mathrm{kcr}}$ & $\Phi_{i}^{\text {sub }}$ & $\Phi^{P r}$ \\
\hline$\lambda$ & $D_{m+2}$ & 2 & $A_{1}^{2}$ & $D_{m+1}$ & $B_{m}$ \\
$\mu$ & $D_{2 m}$ & $m$ & $A_{1}^{m}$ & $A_{2 m-1}$ & $C_{m}$ \\
$\nu$ & $D_{2 m+1}$ & $m+1$ & $A_{1}^{m}$ & $A_{2 m}$ & $B C_{m}$ \\
$\varnothing$ & $E_{7}$ & 3 & $A_{1}^{3}$ & $E_{6}$ & $F_{4}$ \\
$\psi$ & $E_{6}$ & 4 & $A_{2}^{2}$ & $D_{4}$ & $G_{2}$
\end{tabular}


This may be checked in each case by means of the standard models of the root systems $\Phi=D_{n} ; E_{7} ; E_{6}$, but for convenience we offer a reasonably unified argument.

Since $M$ is a negative root, $M-\eta(M)$ for $\eta \neq \psi$ and $M-1 / 3$. $\left(M+\eta(M)+\eta^{2}(M)\right)$ for $\eta=\psi$ is not in the subspace spanned by the fundamental roots different from $\eta(M), \eta^{2}(M)$. Hence the multiplicity of an eigenvalue different from 1 of $\eta$ equals the number of $\eta$-orbits of length $>1$ containing a fundamental root. Therefore $\operatorname{dim} P p^{-1}(0)=$ $2 ; m ; m+1 ; 3 ; 4$ and $\operatorname{dim} V=m ; m ; m ; 4 ; 2$ where $V$ is the space of $\eta$-invariant points.

(1.2) Let $\phi_{1}^{\text {sub }}$ be the $\eta$-invariant subsystem of $\Phi$ of type $D_{m-1} ; A_{2 m-1}$; $A_{2 m} ; E_{6} ; D_{4}$ generated by fundamental roots. See $(0.3)$. Then the restriction $\eta \mid \Phi_{1}^{\text {sub }}$ is the usual twisting isometry, and so $F r\left(\bar{\Phi}_{1}^{\text {sub }}\right)$ is a root system of type $B_{m} ; C_{m} ; B C_{m} ; F_{4} ; G_{2}$ which clearly spans $V$.

Let $\Phi_{2}^{\mathrm{sub}}$ be another $\eta$-invariant subsystem of $\Phi$ of the same type as $\phi_{1}^{\text {sub }}$ spanned by roots in the extended diagrams $(0.3)$ if $\gamma=\lambda ; \mu ; \nu$, by $r_{1}, M+r_{2}, r_{3}, r_{4}, r_{5}, r_{6}+r_{7}$ if $\eta=\varphi$ and $b y r_{1}+M, r_{2}+r_{3}, r_{4}, r_{5}+r_{6}$ if $\eta=\psi$.

Now $\operatorname{Pr}\left(\Phi_{2}^{\text {sub }}\right)$ and $\operatorname{Pr}\left(\Phi_{2}^{\text {sub }}\right)$ are root systems of the same type, each of them spanning $V$. We will show that they coincide:

To any root $r \in \operatorname{Pr}\left(\Phi_{1}^{\text {sub }}\right) \cup \operatorname{Pr}\left(\Phi_{2}^{\text {sub }}\right)$ the usual twisting procedure assigns an element $w_{r} \in W(\Phi)$, the reflection group defined by $\Phi$, such that $w_{r}(V)=V$ and $w_{r} \mid V$ is the refection along $r$. Consider the group generated by the elements $w_{r}$,

$$
W^{\prime}=\left\langle w_{r} ; r \in \operatorname{Pr}\left(\bar{\Phi}_{1}^{\mathrm{sub}}\right) \cup \operatorname{Pr}\left(\Phi_{2}^{\mathrm{sil}}\right)\right\rangle
$$

Since $W^{\prime} \subset W^{\prime}(\Phi)$, $W^{\prime}$ and $W^{\prime} \mid V$ are finite. Since $W^{\prime} V$ is a finite refiection group, $\operatorname{Pr}\left(\Phi_{1}^{\text {sub }}\right) \cup \operatorname{Pr}\left(\Phi_{2}^{\text {sub }}\right)$ generate a root system $\Phi^{\prime}$ in $V$.

If $\operatorname{Pr}\left(\Phi_{1}^{\mathrm{sub}}\right) \neq \operatorname{Pr}\left(\Phi_{2}^{\mathrm{sub}}\right)$, there is a proper inclusion $\operatorname{Pr}_{r}\left(\Phi_{2}^{\mathrm{sub}}\right) \subset \Phi^{\prime}$. But the only inclusions between indecomposable root systems we have to consider are $B_{m} \subset B C_{m}, C_{m} \subset B C_{m}, B_{4} \subset F_{4}, C_{4} \subset F_{4}$. The first two possibilities are excluded by the fact that $\operatorname{Pr}\left(\Phi_{1}^{\text {sub }}\right)$ and $\operatorname{Pr}\left(\Phi_{2}^{\text {sui }}\right)$ consist of the same types of roots. The two last possibilities $(\eta=\lambda ; \because, m=4)$ may then be excluded by regarding $\eta=\lambda$; $\mu$ as a restriction to an $\eta$-invariant $D_{6} ; D_{8}$-subsystem of $\Phi=D_{m+2} ; D_{2 m}$ with $m>4$. Hence $\operatorname{Pr}\left(\Phi_{1}^{\mathrm{sub}}\right)=\operatorname{Pr}\left(\check{\Phi}_{2}^{\mathrm{sub}}\right)$.

All fundamental roots of $\Phi$ are contained in $\Phi_{1}^{\text {sub }}, \Phi_{2}^{\text {sub }}$ or $\Phi_{-}^{\text {sni }}+\Phi_{2}^{\text {sub }}$. Therefore $\Phi^{\prime}=\operatorname{Pr}(\Phi)-\{0\}$, and so $\Phi^{P r}=\operatorname{Pr}\left(\Phi_{i}^{\text {sub }}\right), \quad i=1,2$. This establishes (0.6) for $i=1,2$, and that $\Phi^{P r}$ is of type $B_{m} ; C_{m} ; B C_{m} ; F_{4} ; G_{2}$ in the cases $\eta=\lambda ; \mu ; \nu ; \varphi ; \psi$ respectively.

The classification of the systems $\Phi^{\mathrm{ker}}=\Phi \cap \operatorname{Pr}^{-1}(0)$ may now be done by the following counting argument:

$\Phi_{2}^{\text {sub }}$ contains $\eta$-orbits of lengths 2 and 3 of the same types as 
$\Phi_{1}^{\text {sub }}$ contains, that are not contained in $\Phi_{1}^{\text {sub }}$. This is obvious when there is only one such type to consider, and for $\eta=\nu\left(M, r_{2 m+1}\right)$ and $\left(M+r_{2}+\cdots+r_{m}, r_{m+1}+\cdots+r_{2 m-1}+r_{2 m+1}\right)$ are examples, with reference to (0.3). Thus one, hence (by application of elements of $W^{\prime}$ ) all short roots and halves of long roots in $\Phi^{P r}$ are projections of more than one $\eta$-orbit of roots in $\Phi$.

$\Phi$ contains $2 m^{2}+6 m+4 ; 8 m^{2}-4 m ; 8 m^{2}+4 m ; 126 ; 72$ roots. $\Phi^{P r}$ contains $2 m ; 2 m^{2}-2 m ; 2 m^{2}-2 m ; 24 ; 6$ short and $2 m^{2}-2 m ; 2 m ; 2 m ; 24 ; 6$ long or mixed roots. So far then, $\operatorname{Pr}^{-1}\left(\Phi^{P r}\right)$ accounts for $2 m \cdot 4+$ $\left(2 m^{2}-2 m\right) \cdot 1 ;\left(2 m^{2}-2 m\right) \cdot 4+2 m \cdot 1 ;\left(2 m^{2}-2 m\right) \cdot 4+2 m \cdot 5 ; 24 \cdot 4+24 \cdot 1$; $6 \cdot 6+6 \cdot 1$ roots of $\Phi$, and $4 ; 2 m ; 2 m ; 6 ; 30$ remain to be placed.

But for $\eta=\psi$, all 30 roots cannot be in $\operatorname{Pr}^{-1}(0)$, since no root system of dimension $\leqq 4$ has 30 roots. So in this case $3 \eta$-orbits of length 3 have the same projection. Now the remaining $4 ; 2 m ; 2 m ; 6 ; 12$ roots are so few that none of them can be in $\operatorname{Pr}^{-1}\left(\Phi^{P r}\right)$. Hence they form a root system $\Phi^{\mathrm{ker}} \subset \operatorname{Pr}^{-1}(0)$.

$W\left(\Phi^{\text {ker }}\right)$ permutes the roots inside each $\operatorname{Pr}^{-1}(r), r \in \Phi^{P r}$. Since $\Phi \cap \operatorname{Pr}^{-1}\left(\Phi^{P r}\right)$ generates $\Phi$ (it contains $\left.\Phi_{1}^{\text {sub }} \cup \Phi_{2}^{\text {sub }}\right), W\left(\Phi^{\mathrm{ker}}\right)$ has a faithful permutation representation on $\Phi \cap \operatorname{Pr}^{-1}\left(\Phi^{P r}\right)$. For

$$
\eta \neq \psi, \quad \eta \mid \operatorname{Pr}^{-1}(0)=-1
$$

so $\eta$ centralizes $W\left(\Phi^{\mathrm{ker}}\right)$ and $W\left(\Phi^{\mathrm{ker}}\right)$ permutes the $\eta$-orbits inside each $\operatorname{Pr}^{-1}(r) \cap \Phi, r \in \Phi^{P r}$. Hence the elements of $W\left(\Phi^{\mathrm{ker}}\right)$ have orders 1,2 or (conceivably) 4 if $\eta \neq \psi$. For $\eta=\psi$ we remark that the only onerootlength systems with 12 roots and dimension $\leqq 4$ are $A_{2} \times A_{2}$ and $A_{3}$. But if $\Phi^{\mathrm{ker}}=A_{3}$, the restriction of $\psi$ to the subspace spanned by $\Phi^{\mathrm{ker}}$ must have an eigenvalue equal to 1 . Hence $\Phi^{\mathrm{ker}}=A_{2} \times A_{2}$. Consequently the only possibilities are $\Phi^{\mathrm{ker}}=A_{1}^{2} ; A_{1}^{m} ; A_{1}^{m} ; A_{1}^{3} ; A_{2}^{2}$ in the five cases $\eta=\lambda ; \mu, \nu ; \varphi ; \psi$ respectively.

This establishes (1.1).

(1.3) In the sequel, $\Omega$ will denote an arbitrary component of $\Phi^{\mathrm{ker}}$. By (1.1) $\Omega$ is of type $A_{1}$ if $\eta \neq \psi$ and of type $A_{2}$ if $\eta=\psi$.

The discussion above shows that any root in $\Phi$ belongs to one of five classes: $K, L, 4,5,9$ defined as follows:

$K:$ a root in $\Phi^{\mathrm{ker}}$.

$L:$ an $\eta$-invariant root, i.e., a long root in $\Phi^{P r}$.

4: a root in an $\eta$-orbit of two orthogonal roots, i.e., belonging

to a set $\operatorname{Pr}^{-1}(r) \cap \Phi$ of 4 roots, $r \in \Phi^{P r}$ a short root, $\eta \neq \psi$.

5: a root in an $\eta$-orbit of two roots making a $120^{\circ}$-angle, i.e., in a set $\operatorname{Pr}^{-1}(\{s, 2 s\}) \cap \Phi$ of 5 roots, $s \in \Phi^{P r}$ a half-root, $\eta=\nu$.

9: a root in an $\eta$-orbit of three orthogonal roots, i.e., in a set $\operatorname{Pr}^{-1}(r) \cap \Phi$ of 9 roots, $r \in \Phi^{P r}$ a short root, $\eta=\psi$. 
Let $\eta \neq \psi$ and let $r, s \in \Phi^{P r}, r$ a short root, $s$ a half-root. Then $\operatorname{Pr}^{-1}(\{r,-r\}) \cap \Phi$ and $\operatorname{Pr}^{-1}(\{2 s, s,-s,-2 s\}) \cap \Phi$ generate root systems $X$ and $Y$ of dimensions $\leqq 3$ with $\geqq 8$ and $\geqq 10$ roots respectively. Hence $Y$ is an $A_{3}$-system. $X$ must be an $A_{2} \times A_{1^{-}}$or an $A_{3}$-system, but the first is impossible, since the 8 roots would have to form 4 $\eta$-orbits of orthogonal roots. $X$ contains four $K$-roots and $Y$ contains two. It is useful to show $\eta \mid X$ and $\eta \mid Y$ in two figures. Only a positive subsystem is shown.
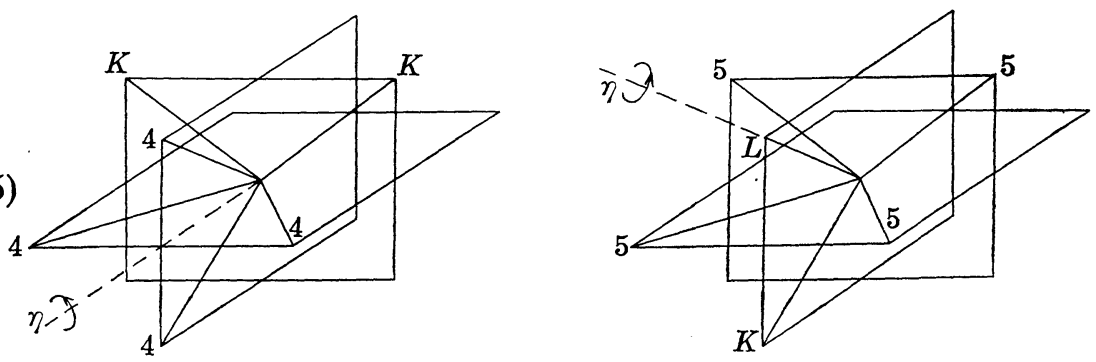

We now introduce a new ordering of the roots in $\Phi$, with the property that if $\operatorname{Pr}(r)=\operatorname{Pr}(s) \neq 0, r, s \in \Phi$ and $r$ is positive in the new ordering, then also $s$ is positive. This is done by selecting positive systems $\Phi^{\text {Pr+ }}$ in $\Phi^{\text {Pr }}$ and $\Phi^{\mathrm{ker}-\mathrm{i}}$ in $\Phi^{\mathrm{ker}}$ (i.e., in each component $\Omega$ of $\left.\Phi^{\mathrm{ker}}\right)$, and defining

$$
\Phi^{+}=\left(\operatorname{Pr}^{-1}\left(\Phi^{P r+}\right) \cap \Phi\right) \cup \Phi^{\mathrm{ker}+} .
$$

$\Phi^{+}$spans a convex cone containing one half of $\Phi$, hence $\Phi^{+}$is a positive system in $\Phi$, different from the one defining the original fundamental roots in (0.3).

The fundamental roots in $\Phi^{+}$span the extremelines of the cone. Therefore the numbers of fundamental roots of types $K, L, 4,5,9$ may be read off from (1.1). They are listed in Table (1.7):

\begin{tabular}{cccccc}
$\eta$ & $K$ & $L$ & 4 & 5 & 9 \\
\hline$\lambda$ & 2 & $m-1$ & 1 & 0 & 0 \\
$\mu$ & $m$ & 1 & $m-1$ & 0 & 0 \\
$\nu$ & $m$ & 0 & $m-1$ & 2 & 0 \\
$\varphi$ & 3 & 2 & 4 & 0 & 0 \\
$\psi$ & 4 & 1 & 0 & 0 & 1
\end{tabular}

From (1.5) we see that in the Dynkin diagram with the fundamental roots of $\Phi^{+}$as nodes, a 4-root or a 5-root always occur in a subdiagram

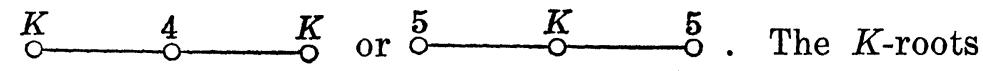
and any $L$-root form a subdiagram of type $\Phi^{\text {ker }} \times A_{1}$. 
These remarks together with (1.7) suffice to determine the new Dynkin diagrams by simple conbinatorial arguments. They are:

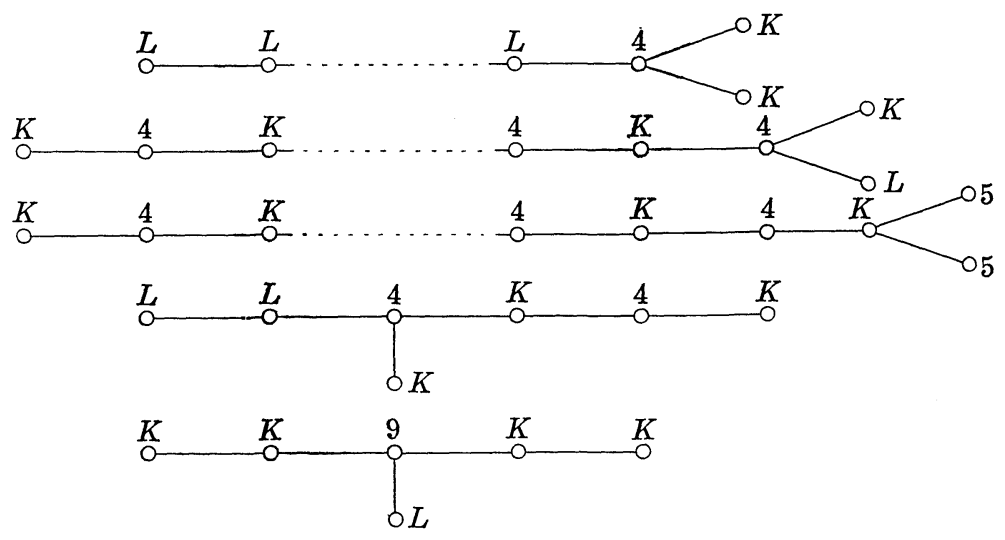

Now we make the following observations:

(1.9) If $\eta \neq \psi$, a reflection along a $K$-root in (1.5) interchanges the two $\eta$-orbits with the same projection.

(1.10) If $\eta=\psi,(1.8)$ shows that the 18 roots in $\operatorname{Pr}^{-1}(\{r,-r\}) \cap \Phi$, $r \in \Phi^{P r}$ a short root, generate an $\psi$-invariant $A_{5}$-system

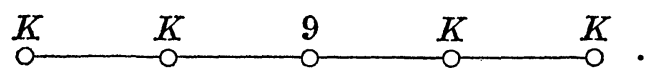

$\psi$ rotates the two $A_{2}$-components of $\Phi^{\mathrm{ker}} 120$ degrees. The centralizer of $\psi$ in $W\left(\Phi^{\mathrm{ker}}\right)$ is isomorphic to $Z_{3} \times Z_{3}$, and it is easily checked that a subgroup permutes the three $\psi$-orbits in $\operatorname{Pr}^{-1}(r) \cap \Phi$ cyclically.

Any subsystem $\Phi_{i}^{\text {sub }} \subset \Phi$ which satisfies (0.6) is specified by the selection of one $\eta$-orbit inside each $\operatorname{Pr}^{-1}(r) \cap \Phi$ where $r \in \Phi^{P r+}$ is a fundamental short root or half-root. The distribution of $K$-roots in (1.8) together with (1.9) and (1.10) now show that there are exactly $N$ such subsystems, with

$$
N=2 ; 2^{m-1} ; 2^{m} ; 4 ; 3 \text { for } \eta=\lambda ; \mu ; \nu ; \phi ; \psi \text { respectively } .
$$

In the sequel $\eta$-invariant subsystems of the types shown in (1.5) and described in (1.10) play an important role. We therefore introduce the following notation:

(1.12) The $\eta$-invariant $A_{3}$-system with four $K$-roots and eight 4-roots, $\eta \neq \psi$, will be called a K4K-system. The $\eta$-invariant $A_{3}$-system with two K-roots, two L-roots, and eight 5-roots, $\eta=\nu$, will be called a 5K5-system. The $\eta$-invariant $A_{5}$-system of $(1.10)$ with twelve K-roots and eighteen 9-roots, $\eta=\psi$, will be called a KK9KK-system.

Clearly there are one-to-one correspondences between $K 4 K$-systems and pairs $\{r,-r\}, r$ short, $\eta \neq \psi$, between $5 K 5$-systems and pairs $\{s,-s\}, s$ a half-root, $\eta=\nu$, between $K K 9 K K$-systems and pairs 
$\{r,-r\}, r$ short, $\eta=\psi$. See (1.5) and (1.10).

2. The Lie algebras and the Chevalley groups. In the complex simple Lie algebra $\mathscr{L}(\Phi)$ defined by the root system $\Phi$, let

$$
\left\{X_{r} ; r \in \Phi\right\} \cup\left\{H_{r_{i}} ; r_{i} \text { fundamental in } \Phi\right\}
$$

be a Chevalley basis, $r_{1}, r_{2}, \cdots$ being the original fundamental roots of (0.3).

From the theory of Lie algebras we recall a few facts:

(2.2) The selection of a Cartan algebra in $\mathscr{L}(\Phi)$ determines the rootspaces, and inside these the selection of

$$
\left\{X_{r_{i}} ; r_{i} \text { fundamental in } \Phi\right\}
$$

is arbitrary and determines the Chevalley basis (2.1) except for signs. For every $r \in \Phi, X_{r}$ determines $X_{-r}$ through the equation

$$
\text { [[ } \left.\left.X_{r}, X_{-r}\right], X_{r}\right]=2 X_{r}
$$

We introduce some subalgebras of $\mathscr{L}(\Phi)$ :

$$
\begin{aligned}
& \mathscr{L}\left(\Phi_{i}^{\mathrm{sub}}\right)=\left\langle X_{r} ; r \in \Phi_{i}^{\mathrm{sub}}\right\rangle, 1 \leqq i \leqq N \\
& \mathscr{L}\left(\Phi^{\mathrm{ker}}\right)=\left\langle X_{r} ; r \in \Phi^{\mathrm{ker}}\right\rangle
\end{aligned}
$$

$\mathscr{L}(\Omega), \mathscr{L}(K 4 K), \mathscr{L}(5 K 5), \mathscr{L}(K K 9 K K)$ denote four types of subalgebras generated by the $X_{r}$-elements with $r$ in an $\Omega$-, $K 4 K-, 5 K 5$ - or $K K 9 K K$-system. See (1.3) and (1.12).

(2.4) Lemma. For any $i, 1 \leqq i \leqq N,\left\langle\mathscr{L}\left(\Phi_{i}^{\mathrm{sub}}\right), \mathscr{L}\left(\Phi^{\mathrm{ker}}\right)\right\rangle=\mathscr{L}(\Phi)$.

Proof. Observe that for any $i, 1 \leqq i \leqq N$, and any given $K 4 K$-, $5 K 5$ - or $K K 9 K K$-system and with $\Phi^{+}$as in (1.6),

$$
\begin{array}{ll}
\Phi^{+} \cap \Phi_{i}^{\text {sub }} \cap K 4 K & \text { consists of one } \eta \text {-orbit of two orthogonal roots, } \\
\Phi^{+} \cap \Phi_{i}^{\text {sub }} \cap 5 K 5 & \text { consists of three roots, } r, \eta(r), r+\eta(r), \\
\Phi^{+} \cap \Phi_{i}^{\text {sub }} \cap K K 9 K K & \begin{array}{l}
\text { consists of one } \eta \text {-orbit of three orthogonal } \\
\text { roots. }
\end{array}
\end{array}
$$

This follows from (0.6) and the Definition (1.12). Taking Lie-products inside each $\mathscr{L}(K 4 K), \mathscr{L}(5 K 5)$, and $\mathscr{L}(K K 9 K K)$ one obtains $\pm X_{r}$ for all $r \notin \Phi^{\text {ker }} \cup \Phi_{i}^{\text {sub }}$.

(2.5) Definition. Let $\eta(=\lambda ; \mu ; \nu ; \varphi ; \psi)$ denote an $\mathscr{L}(\Phi)$-automorphism such that

$$
\eta: X_{r_{i}} \longrightarrow X_{\eta\left(r_{i}\right)}, \quad X_{-r_{i}} \longrightarrow X_{-\eta\left(r_{i}\right)}
$$


where $r_{1}, r_{2}, \cdots$ are the original fundamental roots from (0.3).

This defines an $\mathscr{L}(\Phi)$-automorphism by the isomorphism theorem for Lie algebras, but because of the facts (2.2) it is necessary to make the following remark:

(2.6) The Definition (2.5) is ambiguous, given the elements $X_{r_{1}}, X_{r_{2}}$, ..., pending the choice between two possibilities for $X_{M}$ in the Chevalley basis, $M$ being as in (0.3).

(2.7) Lemma. All the subalgebras of (2.3) are $\eta$-invariant.

Proof. This follows from (2.5) and the fact that all the root subsystems $\Phi_{i}^{\text {sub }}(1 \leqq i \leqq N), \Phi^{\mathrm{ker}}, \Omega \subset \Phi^{\mathrm{ker}}, K 4 K, 5 K 5, K K 9 K K$ of $\Phi$ are $\eta$-invariant (for the isometry $\eta$ ).

(2.8) Lemma. $\eta$ is expressed by an integral matrix with respect to the Chevalley basis (2.1).

Proof. The $\eta$-images of the Chevallay basis elements different from $H_{y-1(M)}$ of (2.1) are clearly in the $Z$-span of that basis. But also $\eta\left(H_{\eta^{-1}(M)}\right)=\eta\left[X_{\eta^{-1}(M)}, X_{-\eta^{-1}(M)}\right]=\left[X_{M}, X_{-M}\right]$ belongs to this $Z$-span, by a fundamental property of a Chevalley basis.

Clearly, for every $r \in \Phi$ either $\eta\left(X_{r}\right)$ or $-\eta\left(X_{r}\right)$ belongs to the Chevalley basis. Hence the $\mathscr{L}(\Phi)$-automorphism $\eta$ has order $|\eta|=$ $2 ; 2 ; 2 ; 2 ; 3$ if and only if $\eta\left(X_{M}\right)=X_{\eta(M)}$. The lemmas (2.10)-(2.13) show that this is actually so, and also settles the question of how the choice of $X_{M}$ affects the $\mathscr{L}(\Phi)$-automorphism $\eta$. See (2.6).

(2.9) Two $\mathscr{L}(\Phi)$-automorphisms $\alpha$ and $\beta$ will be called equivalent if there exists an $\mathscr{L}(\Phi)$-automorphism $\gamma$ such that

$$
\gamma\left(X_{r}\right)= \pm X_{r} \text { for all } r \in \Phi \text { and } \alpha=\gamma \cdot \beta \cdot \gamma^{-1}
$$

(2.10) Lemma. In case $\eta=\psi$, the two choices of $X_{M}$ give two equivalent $\mathscr{L}\left(E_{6}\right)$-automorphisms.

Proof. Call the two $\mathscr{L}\left(E_{6}\right)$-automorphisms $\psi_{1}$ and $\psi_{2}$, so that

$$
\psi_{1}\left(X_{r_{6}}\right)=-\psi_{2}\left(X_{r_{6}}\right) \text {. See }(0.4)
$$

Define $\gamma$ by

$$
\gamma\left(X_{r_{4}}\right)=-X_{r_{4}}, \quad \gamma\left(X_{r_{i}}\right)=X_{r_{i}} \text { for } i \neq 4 .
$$

The highest root in $E_{6}$ with respect to the ordering of $(0.3)$ is 
604

EIVIND STENSHOLT

$$
-M=\sum k_{i} \cdot r_{i}=2 r_{1}+r_{2}+2 r_{3}+3 r_{4}+2 r_{5}+r_{6},
$$

so, in particular, $k_{4}$ is odd. Expressing $X_{-M}$ as a repeated Lie-product of the $X_{r_{i}}$-elements we see that

$$
\gamma\left(X_{-M}\right)=-X_{-M}, \text { hence by (2.2) } \gamma\left(X_{M}\right)=-X_{M} .
$$

It is now easily checked that $\gamma \cdot \psi_{1} \cdot \gamma^{-1}=\psi_{2}$.

Because of (2.10) it is not necessary to distinguish between the two cases, and $\psi$ will mean any of the two $\mathscr{L}\left(E_{6}\right)$-automorphisms of (2.5).

(2.11) Lemma. $\psi^{3}=1$ as an $\mathscr{L}\left(E_{6}\right)$-automorphism.

Proof. As remarked above it suffices to show that $\psi\left(X_{M}\right)=X_{r_{2}}$. Suppose contrariwise $\psi\left(X_{M}\right)=-X_{r_{2}}$. Then $\psi^{3}\left(X_{r_{i}}\right)=X_{r_{i}}$ for $i=1$, $3,5,4$ and $\psi^{3}\left(X_{r_{i}}\right)=-X_{r_{i}}$ for $i=2,6$ and $\psi^{3}\left(X_{M}\right)=-X_{M}$. Express $X_{-M}$ as in the preceding proof. Since $k_{2}+k_{6}$ is even,

$$
\psi^{3}\left(X_{-M}\right)=1\left(X_{-M}\right)=X_{-M} \text {, hence by }(2.2) \psi^{3}\left(X_{M}\right)=X_{M},
$$

which is a contradiction. Consequently $\psi^{3}=1$.

(2.12) Lemma. For $\eta \neq \psi, \eta^{2}=1$ as an $\mathscr{L}(\Phi)$-automorphism, for both choices of $X_{M}$.

Proof. Let $\Phi_{1}^{\text {sub }}$ be as in (1.2). Clearly $\eta^{2} \mid \mathscr{L}\left(\Phi_{1}^{\text {sub }}\right)=1$, and so by (2.4) it suffices to prove that $\eta^{2} \mid \mathscr{L}\left(\Phi^{\mathrm{ker}}\right)=1$.

For a given $K$-root $r \in \Phi$, there exist $a, b= \pm 1$ such that $\eta\left(X_{r}\right)=a X_{-r}$ and $\eta\left(X_{-r}\right)=b X_{r}$. The equation in (2.2) becomes $\left[\left[a X_{-r}, b X_{r}\right], a X_{-r}\right]=2 a X_{-r}$, which shows that $a b=1, a=b$ and consequently $\eta^{2}\left(X_{r}\right)=X_{r}$.

(2.13) Lemma. For $\eta \neq \psi$, the two choices of $X_{M}$ give two inequivalent $\mathscr{L}(\Phi)$-automorphisms $\eta^{+}$and $\eta^{-}$(see (2.9)) where

$$
\eta^{+}\left(X_{r}\right)=X_{-r} \text { and } \eta^{-}\left(X_{r}\right)=-X_{-r} \text { for all } K \text {-roots } r \in \Phi \text {. }
$$

Proof. Let $\Phi_{1}^{\text {sub }}$ be as in (1.2). Since $\eta \mid \mathscr{L}\left(\Phi_{1}^{\text {sub }}\right)$ is independent of the choice of $X_{M}$, the two choices must give different restrictions $\eta \mid \mathscr{L}\left(\Phi^{\mathrm{ker}}\right)$ because of (2.4).

Let $r, s, t$ be fundamental roots of a $K 4 K$-system, $r$ and $t$ being $K$-roots and $s$ a 4-root. See (1.4), (1.5), (1.12). In the standard model for $\mathscr{L}(K 4 K)$ of $4 \times 4$-matrices, let $E_{12}, E_{23}, E_{34}$ represent $X_{r}, X_{s}, X_{t}$ respectively. Identifying $\mathscr{L}(K 4 K)$ with this matrix algebra we have 
$\eta: \quad E_{12}, E_{34}, E_{13}, E_{31}, E_{14}, E_{41} \longrightarrow a E_{21}, b E_{43}, c E_{24}, c E_{42}, d E_{23}, d E_{32}$

for certain $a, b, c, d= \pm 1$. By (2.12), $\eta^{2}=1$ and so

$$
\begin{aligned}
& c E_{24}=\eta\left(E_{13}\right)=\eta\left(\left[E_{12}, E_{23}\right]\right)=\left[a E_{21}, d E_{14}\right]=a d E_{24} \\
& d E_{41}=\eta\left(E_{32}\right)=\eta\left(\left[E_{34}, E_{42}\right]\right)=\left[b E_{43}, c E_{31}\right]=b c E_{41} .
\end{aligned}
$$

Hence $c d=a b c d, a b=1, a=b$ and so $\eta\left(X_{r}\right)=a X_{-r}, \eta\left(X_{t}\right)=a X_{-t}$. Applying this result to all $K 4 K$-subsystems defined by the Dynkin diagrams of (1.8), we find that $\eta\left(X_{r}\right)=a X_{-r}$ for all $K$-roots $r \in \Phi$ with $a= \pm 1$ independent of $r$.

The next result shows that the restrictions $\eta \mid \mathscr{L}\left(\Phi_{i}^{\text {sub }}\right), 1 \leqq i \leqq N$, are related to each other in a sense similar to (2.9):

(2.14) Lemma. For every pair $(i, j)$ such that $1 \leqq i<j \leqq N$, there

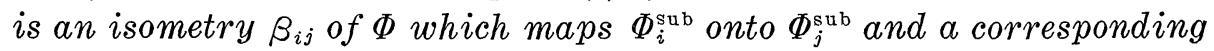
isomorphism (also denoted $\left.\beta_{i j}\right)$ of $\mathscr{L}\left(\Phi_{i}^{\mathrm{sub}}\right)$ onto $\mathscr{L}\left(\Phi_{j}^{\mathrm{sub}}\right)$, i.e., $\beta_{i j}\left(X_{r}\right)=$ $\pm X_{\beta_{i j}(r)}$ for every $r \in \Phi_{j}^{\mathrm{sub}}$, with the additional property:

$$
\beta_{i j} \cdot \eta\left|\mathscr{L}\left(\Phi_{i}^{\mathrm{sub}}\right) \cdot \beta_{i j}^{-1}=\eta\right| \mathscr{L}\left(\Phi_{j}^{\mathrm{sub}}\right) \text {. }
$$

Proof. The subsystems $\Phi_{i}^{\text {sub }}, 1 \leqq i \leqq N$ are conjugates under $W\left(\Phi^{\mathrm{ker}}\right)$ (see the remarks in connection with (1.11)). Let $\gamma \in W\left(\Phi^{\mathrm{ker}}\right)$ be such that $\gamma\left(\Phi_{i}^{\text {sub }}\right)=\Phi_{j}^{\text {sub }}$. Let $\gamma$ also denote a corresponding $\mathscr{L}(\Phi)$ automorphism, such that $\gamma\left(X_{r}\right)= \pm X_{r(r)}$ for all $r \in \Phi$. Then

$$
\gamma \cdot \eta\left|\mathscr{L}\left(\Phi_{i}^{\mathrm{sub}}\right) \cdot \gamma^{-1}=\eta\right| \mathscr{L}\left(\Phi_{j}^{\mathrm{sub}}\right) \cdot \beta
$$

where $\beta$ is an $\mathscr{L}\left(\Phi_{j}^{\text {sub }}\right)$-automorphism such that $\beta\left(X_{r}\right)= \pm X_{\gamma}$ for $r \in \Phi_{j}^{\text {sub }}$, since $\gamma \eta \gamma^{-1}=\eta$ as isometries.

Now, if $\eta(r)=r$, i.e., if $r$ is an $L$-root, then $r \in \Phi_{i}^{\text {sub }} \cap \Phi_{j}^{\text {sub }}$ and $\gamma(r)=r$. Hence by $(2.15) \eta\left(X_{r}\right)=\gamma \cdot \eta \cdot \gamma^{-1}\left(X_{r}\right)=\eta \cdot \beta\left(X_{r}\right)$ which implies

$$
\beta\left(X_{r}\right)=X_{r} \text { if } r \text { is an } L \text {-root. }
$$

All mappings $\alpha: X_{r} \rightarrow \pm X_{r}$ where $r$ belongs to the fundamental system in $\Phi^{+} \cap \Phi_{j}^{\text {sub }}$ extend to $\mathscr{L}\left(\Phi_{j}^{\text {sub }}\right)$-automorphisms. Hence for a suitable $\mathscr{L}\left(\Phi_{j}^{\text {sub }}\right)$-automorphism $\alpha$, because of (2.16),

$$
\alpha \cdot\left(\eta \mid \mathscr{L}\left(\Phi_{j}^{\text {sub }}\right) \cdot \beta\right) \cdot \alpha^{-1}=\eta \mid \mathscr{L}\left(\Phi_{j}^{\text {sub }}\right) .
$$

Now $\beta_{i j}=\alpha \gamma$ meets the requirement of (2.14).

(2.17) Let $G F(q)$ be the finite field with $q$ elements, and let $\theta$ be the automorphism $t \rightarrow t^{q}$ of $G F\left(q^{|\eta|}\right)$ over $G F(q)$.

(2.18) Let $\mathscr{L}(\Phi, q)$ be the Lie algebra $\mathscr{L}(\Phi)_{z} \otimes G F(q)$ over the field $G F(q), \mathscr{L}(\Phi)_{z}$ being the Z-span of the Chevalley basis (2.1). $X_{r} \otimes 1$ is for short denoted $X_{r}, r \in \Phi$. Let $\Phi(q)$ be the Chevalley group with generators 


$$
x_{r}(t)=\exp \left(t \cdot \rho\left(X_{r}\right)\right), \quad t \in G F(q), \quad r \in \Phi,
$$

$\rho$ being a representation of $\mathscr{L}(\Phi, q)$.

We introduce some subgroups of $\Phi(q) . \mathscr{X}_{r}$ is the rootgroup defined by $r \in \Phi$, and:

$$
\begin{aligned}
& \mathscr{\Psi}_{i}^{\text {sub }}(q)=\left\langle\mathscr{X}_{r} ; r \in \Phi_{i}^{\text {sub }}\right\rangle, \quad 1 \leqq i \leqq N \\
& \Phi^{\text {ker }}(q)=\left\langle\mathscr{X}_{r} ; r \in \Phi^{\mathrm{ker}}\right\rangle .
\end{aligned}
$$

$\Omega(q), K 4 K(q), 5 K 5(q), K K 9 K K(q)$ denote four types of subgroups generated by the rootgroups $\mathscr{C}_{r}$ with $r$ in an $\Omega-, K 4 K-, 5 K 5$ or $K K 9 K K$-system. See (1.3) and (1.12).

$$
\mathscr{Y}_{r}=\left\langle\mathscr{Z}_{s} ; s \in \operatorname{Pr}^{-1}(r) \cap \Phi\right\rangle, r \in \Phi^{P r} \text {. }
$$

The structure of $\mathscr{V}_{r}$ depends on $r$ as follows:

$r$ a long root: $r=s, \mathscr{Y}_{r}=\mathscr{X}_{s}, s$ an $L$-root in $\Phi$.

$r$ a short root: $\mathscr{V}_{r}$ is a direct product of $|\eta|^{2}$ rootgroups $\mathscr{X}_{s}$ defined by $|\eta|^{2}$-roots $s \in \operatorname{Pr}^{-1}(r) \cap \Phi$.

$r$ a half-root: Here $\eta=\nu$ and $\mathscr{Y}_{r}$ is a nondirect product of 4 rootgroups $\mathscr{X}_{s}$ defined by 5-roots $s \in \operatorname{Pr}^{-1}(r) \cap \Phi$ and $\mathscr{X}_{2 r}$, $2 r \in \Phi \cap \Phi^{P r}$ being an $L$-root.

(2.21) Lemma. The subgroup $\left\langle\mathscr{Y}_{r}, \mathscr{Y}_{-r}\right\rangle, r \in \Phi^{P r}$, is of the type $A_{1}(q)$; $K 4 K(q) ; 5 K 5(q) ; K K 9 K K(q)$ for $r$ long; $r$ short and $\eta \neq \psi ; r$ a halfroot and $\eta=\nu ; r$ short and $\eta=\psi$ respectively.

Proof. With Chevalley's commutator formulas one easily obtains a set of generators for these groups.

Because of (2.8) $\eta$ may be regarded as an $\mathscr{L}(\Phi, q)$-automorphism and we proceed to define a corresponding $\Phi\left(q^{|\eta|}\right)$-automorphism:

(2.22) Definition. $\eta$ will also denote the $\Phi\left(q^{|\eta|}\right)$-automorphism given by

$$
\eta: x_{r}(t) \longrightarrow \exp \left(\theta(t) \cdot \rho\left(\eta\left(X_{r}\right)\right)\right)=\exp \left(t^{q} \cdot \rho\left( \pm X_{\eta(r)}\right)\right)=x_{\eta(r)}\left( \pm t^{q}\right)
$$

The special cases are denoted $\eta=\lambda ; \mu ; \nu ; \varphi ; \psi$, occasionally subdivided into $\eta^{+} ; \eta^{-}=\lambda^{+} ; \lambda^{-} ; \mu^{+} ; \mu^{-} ; \nu^{+} ; \nu^{-} ; \varphi^{+} ; \varphi^{-}$. See (2.13).

(2.23) Lemma. All the subgroups of (2.19) are $\eta$-invariant.

Proof. This is so because the sets of roots which support these groups are $\eta$-invariant. 
(2.24) Lemma. The $\Phi\left(q^{|\eta|}\right)$-automorphism $\eta$ has order $|\eta|=2 ; 2 ; 2 ; 2 ; 3$ in the cases $\eta=\lambda ; \mu ; \nu ; \varphi ; \psi$ respectively, independently of whether $\eta=\eta^{+}$or $\eta=\eta^{-}$.

Proof. By (2.5) the sign \pm in (2.22) is $a+$ if $r$ or $-r$ is one of the original fundamental roots $r_{1}, r_{2}, \cdots$ in (0.3). Also

$$
\eta\left(x_{M}(t)\right)=\exp \left(\theta(t) \cdot \rho\left(\eta\left(X_{M}\right)\right)\right)=\exp \left(t^{q} \cdot \rho\left(X_{\eta(M)}\right)\right)=x_{\eta(M)}\left(t^{q}\right)
$$

because of (2.11) and (2.12). Similarly $\eta\left(x_{-M}(t)\right)=x_{-\eta(M)}\left(t^{q}\right)$. This implies (2.24).

(2.25) Lemma. In $\Phi_{i}^{\mathrm{sub}}\left(q^{|\eta|}\right)$ there is a subgroup, denoted ${ }^{|\eta|} \Phi_{i}^{\mathrm{sub}}\left(q^{|\eta|}\right)$, of $\eta$-invariant elements which is isomorphic to the groups in (0.7) for $\eta=\lambda ; \mu ; \nu ; \varphi ; \psi$ respectively, independently of $i, 1 \leqq i \leqq N$, and of whether $\eta=\eta^{+}$or $\eta=\eta^{-}$.

Proof. Because of (2.14), the fixpointgroups of the $N$ restrictions $\eta \mid \Phi_{i}^{\text {sub }}\left(q^{|\eta|}\right), 1 \leqq i \leqq N$, are all isomorphic. Hence it suffices to consider $\eta \mid \Phi_{1}^{\text {sub }}\left(q^{|\eta|}\right), \Phi_{1}^{\text {sub }}$ being as in (1.2). But this restriction is just the automorphism which is the basis for Steinberg's twisting procedure.

3. The restriction $\eta \mid \Omega\left(q^{|\eta|}\right), \Omega \subset \Phi^{\mathrm{ker}}$. Let $M(n, q)$ be the algebra of $n \times n$-matrices over $G F(q)$. Let

$$
R^{+} \subset M\left(2, q^{2}\right) ; \quad R^{-} \subset M\left(2, q^{2}\right) ; \quad S \subset M\left(3, q^{3}\right)
$$

respectively consist of the matrices

$$
\left(\begin{array}{ll}
v^{q} & u^{q} \\
u & v
\end{array}\right) ; \quad\left(\begin{array}{cc}
v^{q} & -u^{q} \\
u & v
\end{array}\right) ;\left(\begin{array}{lll}
z^{q^{2}} & b x^{q^{2}} & a y^{q^{2}} \\
c y^{q} & z^{q} & a x^{q} \\
x & y & z
\end{array}\right)
$$

where $u, v \in G F\left(q^{2}\right), x, y, z \in G F\left(q^{3}\right)$ and $a, b, c= \pm 1$ are constants such that $a b c=1$.

(3.2) Lemma. There exist similarity transformations $\alpha^{+} ; \alpha^{-} ; \beta$ of $M\left(2, q^{2}\right) ; M\left(2, q^{2}\right) ; M\left(3, q^{3}\right)$ respectively such that

$$
\alpha^{+}(M(2, q))=R^{+} ; \quad \alpha^{-}(M(2, q))=R^{-} ; \quad \beta(M(3, q))=S .
$$

Proof. Let $K=G F\left(q^{2}\right) ; G F\left(q^{2}\right) ; G F\left(q^{3}\right)$ and let $U$ be the $K$-vectorspace $G F\left(q^{2}\right)^{2} ; G F\left(q^{2}\right)^{2} ; G F\left(q^{3}\right)^{3}$ in the three respective cases. Let $V$ be the $G F(q)$-subspace of $U$ consisting of the vectors

$$
\left(v^{q}, v\right) ; \quad\left(Z v^{q}, v\right) ; \quad\left(c w^{q}, b w^{q}, w\right)
$$


where $v \in G F\left(q^{2}\right), w \in G F\left(q^{3}\right), b, c$ are as in (3.1) and $Z \in G F\left(q^{2}\right)-G F(q)$ is such that $Z^{q}=-Z^{-1}$. This means that

$$
Z=k^{(t+1 / 2)(q-1)} \text { for } q \text { odd and } Z=k^{t(q-1)} \text { for } q \text { even, }
$$

$t$ being an integer and $\langle k\rangle=G F\left(q^{2}\right)-\{0\}$.

The $K$-span of $V$ is $U$ since the vander Monde determinant $\left|k^{i \cdot q j}\right| \neq 0$, $i, j=0,1 ; 0,1 ; 0,1,2$, in the three cases and $\langle k\rangle=K-\{0\}$.

Clearly $R^{+} ; R^{-}$; $S$ have the same number of elements as $M(2, q)$; $M(2, q) ; M(3, q)$ and it is easily checked that $R^{+} ; R^{-} ; S$ act $G F(q)$ linearly on $V$ by right multiplication.

Now replace the standard $K$-basis of $U$ with a $G F(q)$-basis of $V$. This basis-change defines $\alpha^{+} ; \alpha^{-} ; \beta$.

Since $\Phi^{\mathrm{ker}}\left(q^{|x|}\right)$ is a direct product of groups $\Omega\left(q^{|x|}\right)$ (see (1.3)) and since each $\Omega\left(q^{\left|z_{i}\right|}\right)$ is $\eta$-invariant (see (2.23)), the study of $\eta \mid \Phi^{\operatorname{ker}}\left(q^{\left|\eta_{\eta}\right|}\right.$ ) reduces to the study of $\eta \mid \Omega\left(q^{|\eta|}\right)$. Two cases must be distinguished: $\eta \neq \psi$ with $\Omega$ of type $A_{1}$ and $\eta=\psi$ with $\Omega$ of type $A_{2}$.

(3.3) $\eta \neq \psi$ r $, \Omega=\left\{r,-r^{\circ}\right\}, r$ a $K$-root in $\Phi$.

Let $\gamma: S L\left(2, q^{2}\right) \rightarrow \Omega\left(q^{2}\right)$ be a homomorphism such that

$$
\gamma\left(\begin{array}{ll}
1 & t \\
0 & 1
\end{array}\right)=x_{r}(t), \quad \gamma\left(\begin{array}{ll}
1 & 0 \\
t & 1
\end{array}\right)=x_{-r}(t)
$$

Then, by (2.22)

$$
\gamma\left(\begin{array}{ll}
1 & t \\
0 & 1
\end{array}\right) \stackrel{\eta}{\longrightarrow} \gamma\left(\begin{array}{cc}
1 & 0 \\
a b t^{q} & 1
\end{array}\right), \gamma\left(\begin{array}{ll}
1 & 0 \\
t & 1
\end{array}\right) \stackrel{\eta}{\longrightarrow} \gamma\left(\begin{array}{cc}
1 & a b t^{q} \\
0 & 1
\end{array}\right)
$$

with $a, b= \pm 1$, i.e., in general

$$
\eta \gamma\left(\begin{array}{ll}
x & y \\
u & v
\end{array}\right)=\gamma\left(\left(\begin{array}{ll}
0 & a \\
b & 0
\end{array}\right)\left(\begin{array}{ll}
x^{q} & y^{q} \\
u^{q} & v^{q}
\end{array}\right)\left(\begin{array}{ll}
0 & a \\
b & 0
\end{array}\right)\right)=\gamma\left(\begin{array}{cc}
v^{q} & a b u^{q} \\
a b y^{q} & x^{q}
\end{array}\right) .
$$

Hence we get a set of fixpoints of $\eta \mid \Omega\left(q^{2}\right)$ (all the fixpoints if $\gamma$ is an isomorphism):

$$
\gamma\left(\begin{array}{cc}
v^{q} & a b u^{q} \\
u & v
\end{array}\right), \quad u, v \in G F\left(q^{2}\right)
$$

By (3.2) there exists an $\alpha \in \operatorname{Aut} S L\left(2, q^{2}\right)$ such that

(3.4) $\gamma \alpha S L(2, q)$ is contained in the fixpointgroup of $\eta \mid \Omega\left(q^{2}\right)$.

Note that $a b=1$ for $\eta=\eta^{+}$and $a b=-1$ for $\eta=\eta^{-}$.

(3.5) $\eta=\psi r, \Omega=\{r, \psi(r), r+\psi(r),-r,-\psi(r),-r-\psi(r)\}, r$ a $K$-root in $\Phi$. 
Let $\gamma: S L\left(3, q^{3}\right) \rightarrow \Omega\left(q^{3}\right)$ be a homomorphism such that

$$
\begin{aligned}
& \gamma\left(\begin{array}{lll}
1 & 0 & 0 \\
0 & 1 & t \\
0 & 0 & 1
\end{array}\right)=x_{r}(t), \quad \gamma\left(\begin{array}{lll}
1 & t & 0 \\
0 & 1 & 0 \\
0 & 0 & 1
\end{array}\right)=x_{\psi(r)}(t), \quad \gamma\left(\begin{array}{lll}
1 & 0 & t \\
0 & 1 & 0 \\
0 & 0 & 1
\end{array}\right)=x_{r+\psi(r)}(t) \\
& \gamma\left(\begin{array}{lll}
1 & 0 & 0 \\
0 & 1 & 0 \\
0 & t & 1
\end{array}\right)=x_{-r}(t), \gamma\left(\begin{array}{lll}
1 & 0 & 0 \\
t & 1 & 0 \\
0 & 0 & 1
\end{array}\right)=x_{-\psi(r)}(t), \quad \gamma\left(\begin{array}{lll}
1 & 0 & 0 \\
0 & 1 & 0 \\
t & 0 & 1
\end{array}\right)=x_{-r-\psi(r)}(t) \text {. }
\end{aligned}
$$

Then, by (2.22)

$$
\gamma\left(\begin{array}{lll}
1 & 0 & 0 \\
0 & 1 & t \\
0 & 0 & 1
\end{array}\right) \stackrel{\psi}{\longrightarrow} \gamma\left(\begin{array}{ccc}
1 & a b t^{q} & 0 \\
0 & 1 & 0 \\
0 & 0 & 1
\end{array}\right) \stackrel{\psi}{\longrightarrow} \gamma\left(\begin{array}{ccc}
1 & 0 & 0 \\
0 & 1 & 0 \\
b c t^{q^{2}} & 0 & 1
\end{array}\right)
$$

and similarly for the transposed matrices, with $a, b, c= \pm 1$, i.e., in general

$$
\begin{aligned}
\psi \gamma\left(\begin{array}{lll}
f & g & h \\
u & v & w \\
x & y & z
\end{array}\right) & =\gamma\left(\left(\begin{array}{lll}
0 & a & 0 \\
0 & 0 & b \\
c & 0 & 0
\end{array}\right)\left(\begin{array}{lll}
f^{q} & g^{q} & h^{q} \\
u^{q} & v^{q} & w^{q} \\
x^{q} & y^{q} & z^{q}
\end{array}\right)\left(\begin{array}{lll}
0 & 0 & c \\
a & 0 & 0 \\
0 & b & 0
\end{array}\right)\right) \\
& =\gamma\left(\begin{array}{ccc}
v^{q} & a b w^{q} & a c u^{q} \\
a b y^{q} & z^{q} & b c x^{q} \\
a c g^{q} & b c h^{q} & f^{q}
\end{array}\right) .
\end{aligned}
$$

Hence we get a set of fixpoints of $\psi \mid \Omega\left(q^{3}\right)$ (all the fixpoints if $\gamma$ is an isomorphism):

$$
\gamma\left(\begin{array}{ccc}
z^{q^{2}} & a c x^{q^{2}} & b c y^{q^{2}} \\
a b y^{q} & z^{q} & b c x^{q} \\
x & y & z
\end{array}\right), \quad x, y, z \in G F\left(q^{3}\right) .
$$

By (3.2) there exists a $\beta \in$ Aut $S L\left(3, q^{3}\right)$ such that

(3.7) $\quad \gamma \beta S L(3, q)$ is contained in the fixpointgroup of $\psi \mid \Omega\left(q^{3}\right)$.

(3.8) Clearly, the restrictions of $\eta \neq \psi$ to subgroups of types $K 4 K\left(q^{2}\right)$, $5 K 5\left(q^{2}\right)$ and of $\psi$ to subgroups of type $K K 9 K K\left(q^{3}\right)$ can be treated the same way by means of homomorphisms

$$
\begin{aligned}
& S L\left(4, q^{2}\right) \longrightarrow K 4 K\left(q^{2}\right), \quad S L\left(4, q^{2}\right) \longrightarrow 5 K 5\left(q^{3}\right), \\
& S L\left(6, q^{3}\right) \longrightarrow K K 9 K K\left(q^{3}\right) .
\end{aligned}
$$

The matrices are conveniently subdivided into $2 \times 2$-blocks or $3 \times 3$ blocks. Thus the fixpointgroup of $\eta \mid K 4 K\left(q^{2}\right)$ contains the image 
of the matrices (equals in the case of isomorphism):

$$
\left(\begin{array}{cccc}
g^{q} & a b f^{q} & b t^{q} & a h^{q} \\
f & g & h & t \\
b v^{q} & a u^{q} & y^{q} & a b x^{q} \\
u & v & x & y
\end{array}\right) \quad \begin{aligned}
& \text { with determinant 1, } \\
& \text { f }, h, t, u, v, x, y \in G F\left(q^{2}\right) .
\end{aligned}
$$

Similarly $6 \times 6$-matrices with blocks like (3.6) give fixpoints of i $\mid K K 9 K K\left(q^{3}\right)$.

(3.9) Lemma. Let $r \in \Phi^{P r}$ be a short root. Then the fixpointgroup of $\eta \mid \mathscr{Y}_{r}$ has order $q^{\left|\eta^{\prime}\right|^{2}}$.

Proof. There are $|\eta| \eta$-orbits of roots in $\operatorname{Pr}^{-1}(r) \cap \Phi$. With one such orbit we associate as in the usual twisting procedure $q^{|\eta|}$ fixpoints $x_{r}(t) x_{\eta(r)}\left( \pm t^{q}\right)$ if $\eta \neq \psi^{\prime r}$ and $x_{r}(t) x_{\eta(r)}\left( \pm t^{q}\right) x_{\eta^{2}(r)}\left( \pm t^{q^{2}}\right)$ if $\eta=\psi$.

For later reference we need a result which is derived by means of a matrix model for the $5 K 5\left(q^{2}\right)$-groups. We consider the following situation:

(3.10) $\eta=\nu, r \in \Phi^{P r}$ a half-root, $\left\langle\mathscr{V}_{r}, \mathscr{Y}_{-r}\right\rangle$ a $5 K 5\left(q^{2}\right)$-group.

Let $\gamma: S L\left(4, q^{2}\right) \rightarrow\left\langle\mathscr{Y}_{r}, \mathscr{Y}_{-r}\right\rangle$ be a homomorphism such that $\gamma\left(I+E_{i j} t\right)=x_{s}(t)$ where

$s$ is a $K$-root in $\Phi^{+}$if $(i, j)=(2,3)$,

an $L$-root in $\Phi^{+}$if $(i, j)=(1,4)$,

a 5 -root in $\Phi^{+}$if $(i, j)=(1,2),(1,3),(2,4),(3,4)$.

Then

$$
\begin{aligned}
& \gamma\left(I+E_{12} t\right) \stackrel{\nu}{\longrightarrow} \gamma\left(I+E_{24} a t^{q}\right) \\
& \gamma\left(I+E_{34} t\right) \stackrel{\nu}{\longrightarrow} \gamma\left(I+E_{13} b t^{q}\right) \\
& \gamma\left(I+E_{21} t\right) \stackrel{\nu}{\longrightarrow} \gamma\left(I+E_{42} a t^{q}\right) \\
& \gamma\left(I+E_{43} t\right) \stackrel{\nu}{\longrightarrow} \gamma\left(I+E_{31} b t^{q}\right), a, b= \pm 1 .
\end{aligned}
$$

This determines $\eta$ in the $5 K 5\left(q^{2}\right)$-group. One may easily verify that for $A \in S L\left(4, q^{2}\right)$,

$$
\nu \gamma(A)=\gamma\left(X \cdot \theta\left(A^{T}\right)^{-1} \cdot X^{-1}\right)
$$

where

$$
X=\left(\begin{array}{cccc}
0 & 0 & 0 & 1 \\
0 & -a & 0 & 0 \\
0 & 0 & -b & 0 \\
1 & 0 & 0 & 0
\end{array}\right)
$$


$\theta(A)$ is obtained by applying the field automorphism of (2.17) on each entry of $A$, and $T$ denotes transposing. Hence we have:

(3.11) The fixpointgroup of $\eta \mid\left\langle\mathscr{Y}_{r}, \mathscr{Y}_{-r}\right\rangle$ contains a homomorphic image of $S U\left(4, q^{2}\right)$.

Here $a b=1$ for $\nu=\nu^{-}$and $a b=-1$ for $\nu=\nu^{+}$.

(3.12) Lemma. Let $r \in \Phi^{P r}$ be a half-root, $\eta=\nu$. Then the fixpointgroup of $\eta \mid \mathscr{Y}_{r}$ has order $q^{5}$.

Proof. Clearly $\eta \mid \mathscr{Y}_{r}$ is a one-to-one mapping. It then suffices and is easy to verify that the fixpoints of $\eta \mid \mathscr{Y}_{r}$ are the elements

$$
\gamma\left(\begin{array}{cccc}
1 & x & b y^{q} & z \\
0 & 1 & 0 & a x^{q} \\
0 & 0 & 1 & y \\
0 & 0 & 0 & 1
\end{array}\right) \quad \begin{aligned}
& \text { with } x, y, z \in G F\left(q^{2}\right) \text { and } \\
& z+z^{q}=a x x^{q}+b y y^{q}
\end{aligned}
$$

4. The proof of Theorem (0.8).

(4.1) Lemma. Let $\sigma: t \rightarrow x \cdot t^{q}$ be an additive automorphism of $G F\left(q^{n}\right)$, $x \neq 0$ and let $\langle k\rangle=G F\left(q^{n}\right)-\{0\}$. Then $\sigma$ has fixpoints different from 0 if and only if $x \in\left\langle k^{q-1}\right\rangle$. The set of fixpoints is then $t_{0} \cdot G F(q)$, $t_{0} \neq 0$ being an arbitrary fixpoint.

Proof. Let $x=k^{a}, t=k^{b}$. Then $t=\sigma(t)$ is equivalent to the congruence $(q-1) b+a \equiv 0\left(\bmod \left(q^{n}-1\right)\right)$, which implies $a=(q-1) c$ for some $c$ and $b+c \equiv 0\left(\bmod \left(q^{n}-1\right) /(q-1)\right)$. Hence

$$
t=k^{-c+d(q n-1) /(q-1)}, \quad d=0,1, \cdots, q-2
$$

where $\left\langle k^{\left(q^{n}-1\right) /(q-1)}\right\rangle=G F(q)-\{0\}$.

(4.2) Lemma. Let $\gamma$ be a homomorphism of $S L\left(m, q^{n}\right)$ onto a group $H$, and let $\mathscr{Z}_{i j}\left(q^{a}\right)=\gamma\left\{I+E_{i j} t ; t \in G F\left(q^{a}\right)\right\}, i \neq j$ and $a=1$ or $a=n$. Suppose $\alpha \in$ Aut $H$ has the property $\alpha \mathscr{Z}_{i j}(q)=\mathscr{Z}_{2 j}(q)$ for all $(i, j)$. Then also $\alpha \mathscr{Z}_{i j}\left(q^{n}\right)=\mathscr{Z}_{i j}\left(q^{n}\right)$ for all $(i, j)$.

Proof. Let $K_{i j}=\left\{(a, b) ; a \neq b\right.$ and $\left.\left[\mathscr{Z}_{i j}\left(q^{n}\right), \mathscr{Z}_{a b}(q)\right]=1\right\}$. Then it is easily verified that $\mathscr{X}_{i j}\left(q^{n}\right)$ is the only $p$-Sylowgroup ( $p=$ char $G F(q))$ in the centralizer of $\left\langle\mathscr{Z}_{a b}(q) ;(a, b) \in K_{i j}\right\rangle$. This proves the lemma.

In (4.3)-(4.5) we list some facts about automorphisms of Chevalley groups over the field $G F(q)$. 
(4.3) A Chevalley group acts by conjugation transitively on the set of ordered pairs of disjoint $p$-Sylowgroups, $p=\operatorname{char} G F(q)$.

(4.4) An automorphism which normalizes $U$ and $V$ (the products of the rootgroups $\mathscr{X}_{r}$ for $r>0$, resp. $r<0$ ) permutes the rootgroups and can be expressed by diagonal, field and graph automorphisms.

(4.5) Any set $\left\{d_{r} \in G F(q) ; d_{r} \neq 0\right.$ and $r$ is a fundamental root $\}$ determines a diagonal automorphism $d$ where

$$
d: x_{r}(t) \longrightarrow x_{r}\left(d_{r} t\right), \quad x_{-r}(t) \longrightarrow x_{-r}\left(d_{r}^{-1} t\right) .
$$

See Steinberg [4, p. 158].

(4.6) Lemma. $\Phi^{\mathrm{ker}}\left(q^{|r|}\right)$ normalizes $\mathscr{Y}_{r}$ for all $r \in \Phi^{P r}$.

Proof. This is an immediate consequence of Chevalley's commutator formulas (one-rootlength case) since $s \in \Phi^{\mathrm{ker}}$ and $t \in \operatorname{Pr}^{-1}(r) \cap \Phi$ implies $t+s \in \operatorname{Pr}^{-1}(r)$.

Proof of (0.8). Consider first a component $\Omega$ of $\Phi^{\mathrm{ker}}$. By (3.4) and (3.7) there exists a homomorphism

$$
\gamma_{\Omega}: S L\left(|\eta|, q^{|\eta|}\right) \longrightarrow \Omega\left(q^{|\eta|}\right)
$$

such that $\gamma_{\Omega} S L(|\eta|, q)$ is contained in the fixpointgroup of $\eta \mid \Omega\left(q^{|\eta|}\right)$.

An application of (4.3) and (4.4) to each component $\Omega$ of $\Phi^{\text {ker }}$ shows that there exists an inner automorphism $\omega: x \rightarrow g x g^{-1}$ of $\Phi\left(q^{|\eta|}\right)$ defined by an element $g \in \Phi^{\mathrm{ker}}\left(q^{|\eta|}\right)$ such that

$$
\omega \gamma_{\Omega}\left\{I+E_{i j} t ; t \in G F\left(q^{|\eta|}\right)\right\}
$$

is a rootgroup in $\Phi^{\mathrm{ker}}\left(q^{|\eta|}\right)$ for all rootgroups $\left\{I+E_{i j} t\right\}$ of $S L\left(|\eta|, q^{|\eta|}\right)$. Then, for $t \in G F(q)$,

$$
\omega \eta \omega^{-1} \cdot \omega \gamma_{\Omega}\left(I+E_{i j} t\right)=\omega \gamma_{\Omega}\left(I+E_{i j} t\right)
$$

and so, because of Lemma (4.2)

$$
\omega \eta \omega^{-1} \cdot \omega \gamma_{\Omega}\left\{I+E_{i j} t ; t \in G F\left(q^{|\eta|}\right)\right\}=\omega \gamma_{\Omega}\left\{I+E_{i j} t ; t \in G F\left(q^{|\eta|}\right)\right\}
$$

i.e.,

$$
\omega \eta \omega^{-1} \mathscr{X}_{r}=\mathscr{X}_{r} \text { for all } K \text {-roots } r \in \Phi \text {. }
$$

By (4.6) and (2.23)

$$
\omega \eta \omega^{-1} \mathscr{Y}_{r}=\mathscr{Y}_{r} \text { for all } r \in \Phi^{P r} \text {. }
$$

In particular, when $r$ is a long root, this by (2.20) becomes 


$$
\omega \eta \omega^{-1} \mathscr{Z}_{r}=\mathscr{Z}_{r} \text { for all } L \text {-roots } r \in \Phi .
$$

From (4.8) and (4.9) it follows that $\omega \eta \omega^{-1}$ normalizes the subgroups $U$ and $V$ where

$$
\begin{aligned}
& U=\prod_{r \in \phi \mathrm{ker}+} \mathscr{X}_{r} \cdot \prod_{r \in P r+} \mathscr{Y}_{r} \\
& V=\prod_{-r \in \Phi \mathrm{ker}+} \mathscr{X}_{r} \cdot \prod_{-r \in \Phi^{P r+}} \mathscr{Y}_{r} .
\end{aligned}
$$

Hence, by (4.4), $\omega \eta \omega^{-1}$ either normalizes all rootgroups $\mathscr{Z}_{r}, r \in \Phi$, or permutes them according to a graph automorphism. By (1.1) the $K$-roots and $L$-roots span the space of $\Phi$ if and only if $\eta \neq \nu$. Thus we have, by (4.8) and (4.10),

$$
\begin{aligned}
& \omega \eta \omega^{-1} \mathscr{X}_{r}=\mathscr{X}_{r} \text { for all } r \in \Phi \text { if } \eta \neq \nu . \\
& \text { (4.13) } \omega \eta \omega^{-1} \mathscr{Z}_{r}=\mathscr{X}_{\alpha^{\alpha}(r)} \text { for all } r \in \Phi \text { if } \eta=\nu, a=0 \text { or } a=1
\end{aligned}
$$

where $\alpha$ is the graph automorphism of $\Phi=D_{2 m+1}, a$ to be determined later. By (4.7) it is clear that a field automorphism is needed to express $\omega \eta \omega^{-1}$ as in (4.4). Hence by (4.12) and (4.13)

$$
\omega \eta \omega^{-1} x_{r}(t)=x_{s}\left(b_{r} \cdot t^{q}\right) \text { for all } r \in \Phi
$$

with $b_{r} \in G F\left(q^{|\eta|}\right), s=r$ for $\eta \neq \nu, s=\alpha^{a}(r)$ if $\eta=\nu$.

Now design a diagonal automorphism $d$ of $\Phi\left(q^{|\eta|}\right)$ such that

(i) If $r \in \Phi^{+}$is fundamental and $\omega \eta \omega^{-1} \mathscr{Z}_{r}=\mathscr{X}_{r}$, then $\left\{d\left(x_{r}(t)\right)\right.$; $t \in G F(q)\}$ are the $\omega \eta \omega^{-1}$-invariant elements in $\mathscr{Z}_{r}$. This is possible by (4.1) and (4.5).

(ii) If $r \in \Phi^{+}$is fundamental and $\omega \eta \omega^{-1} \mathscr{X}_{r}=\mathscr{X}_{s} \neq \mathscr{X}_{r}$, then $d\left(x_{s}\left(t^{q}\right)\right)=\omega \eta \omega^{-1} d\left(x_{r}(t)\right) . s$ is fundamental in $\Phi^{+}$because the rootgroups are permuted according to a graph automorphisrn, so this may be achieved by adjusting $d_{s}$ in (4.5).

Now it is easily verified that (4.14) is changed into

$$
d^{-1} \omega \eta \omega^{-1} d\left(x_{r}(t)\right)=x_{s}\left(t^{q}\right), \text { for } r \text { or }-r \text { fundamental. }
$$

It remains to determine whether $a=0$ or $a=1$ in (4.13). If $a=0$ however, the fixpointgroup of $\nu$, hence of $\omega \nu \omega^{-1}$, in an arbitrary $5 K 5\left(q^{2}\right)$-group would contain an image of $S L(4, q)$, contradicting (3.11). Hence $a=1$. This proves (0.8).

We remark that similar twists may be performed on the Chevalley groups $F_{4}\left(q^{2}\right)$ and $F_{4}\left(q^{3}\right)$. The long roots in $F_{4}$ form a $D_{4}$-system supporting a subgroup of type $D_{4}\left(q^{2}\right)$ or $D_{4}\left(q^{3}\right)$. Defining $\eta$ as the isometry used for one of the twists of $D_{4}$, the short roots of $F_{4}$ form $\eta$-orbits of roots making $60^{\circ}$-angles and a kernel system of type $A_{1}$ or $A_{2}$. One may check that corresponding $\mathscr{L}\left(F_{4}\right)$-automorphisms of order 2 and 3 exist and proceed as above. The group automorphism 
$\eta$ is conjugate in Aut $F_{4}\left(q^{|\eta|}\right)$ to a field automorphism, and so one obtains embeddings ${ }^{2} D_{4}\left(q^{2}\right) \subset F_{4}(q)$ and ${ }^{3} D_{4}\left(q^{3}\right) \subset F_{4}(q)$.

5. A property of the embeddings (0.10). Let $G$ be the subgroup of the fixpointgroup of $\eta$ in $\Phi\left(q^{|\eta|}\right)$, of the type (0.9). G contains the groups ${ }^{|\eta|} \Phi_{i}^{\text {sub }}\left(q^{|\eta|}\right)$ given in (0.7), because of (2.25).

$G$ may be described in two ways:

First description. We first remark that

(5.1) the $\Phi\left(q^{|\eta|}\right)$-automorphism $\omega^{-1} d$ of (4.15) normalizes $\mathscr{Y}_{r}$ for all $r \in \Phi^{P r}$.

This follows from (4.6) and the definitions of $\omega$ and $d$.

We now introduce new rootgroups $\widetilde{\mathscr{Z}}_{r}$ of $\Phi\left(q^{|\eta|}\right)$ with elements $\widetilde{x}_{r}(t), t \in G F\left(q^{|n|}\right)$ by defining

$$
\tilde{\mathscr{Z}}_{r}=\omega^{-1} d \mathscr{Z}_{r}, \quad \tilde{x}_{r}(t)=\omega^{-1} d\left(x_{r}(t)\right) .
$$

Then, by (4.15)

$$
\eta \widetilde{x}_{r}(t)=\widetilde{x}_{s}\left(t^{q}\right) \text { for } r \text { or }-r \text { fundamental in } \Phi^{+},
$$

$s=r$ if $\eta \neq \nu$ and $s=\alpha(r)$ if $\eta=\nu, \alpha$ being the graph symmetry of $\Phi=D_{2 m+1}$.

This yields a description of $G$ as a group with a $B N$-pair with root system $\Phi^{\text {new }}$ where

$$
\begin{aligned}
& \Phi^{\mathrm{new}}=D_{m+2} ; D_{2 m} ; B_{2 m} ; E_{7} ; E_{6} \quad \text { and } \\
& G \cong D_{m+2}(q) ; D_{2 m}(q) ;{ }^{2} D_{2 m+1}\left(q^{2}\right) ; E_{7}(q) ; E_{6}(q)
\end{aligned}
$$

in the cases $\eta=\lambda ; \mu ; \nu ; \varphi ; \psi$ respectively.

Thus only the third Dynkin diagram of (1.5) must be changed when we move our attention from $\Phi\left(q^{|\eta|}\right)$ to $G$. We repeat the other four diagrams however, indicating by $A, B, C$ some fundamental roots for later reference:
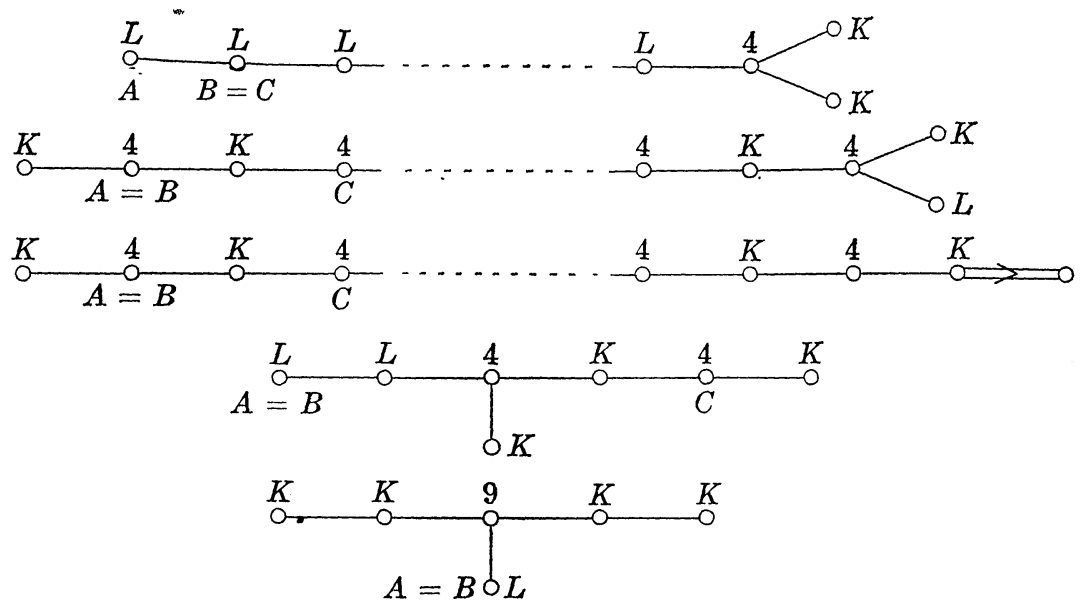
The rootgroups of $G$ will be called $\mathscr{X}_{r}^{\text {now }}, r \in \Phi^{\text {new }}$, with elements $x_{r}^{\text {new }}(t)$. If $\eta \neq \nu$ or $\eta=\nu$ and $r$ is a long root in $\Phi^{\text {new }}$ (i.e., $r \in \Phi \cap \Phi^{\text {new }}$ ), then $\mathscr{X}_{r}^{\text {new }}=\widetilde{\mathscr{X}}_{r} \cap G$ and $x_{r}^{\text {new }}(t)=\widetilde{x}_{r}(t), t \in G F(q)$. If $\eta=\nu$ and $r$ is a short root in $\Phi^{\text {new }}$, then $\mathscr{Z}_{r}^{\text {new }}=\widetilde{\mathscr{Z}}_{s} \cdot \widetilde{\mathscr{X}}_{\alpha(s)} \cap G$ with $s \in \Phi, r=1 / 2(s+$ $\alpha(s))$ and $x_{r}^{\mathrm{new}}(t)=\widetilde{x}_{s}(t) \cdot \widetilde{x}_{\alpha(s)}\left(t^{q}\right), t \in G F\left(q^{2}\right)$. See (5.3) and (5.4).

Second description. $G$ is described in terms of

(i) the subgroups $\Omega\left(q^{|\eta|}\right) \cap G, \Omega$ being a component of $\Phi^{\mathrm{ker}}$. These groups are homomorphic images of $S L(|\eta|, q)$. See (3.4), (3.7).

(ii) the rootgroups of the twisted subgroups ${ }^{|n|} \Phi_{i}^{\text {sub }}\left(q^{|\eta|}\right)$, i.e., the groups

$$
\mathscr{Z}_{r, i}^{\mathrm{sub}}=\mathscr{Y}_{r} \cap \Phi_{i}^{\mathrm{sub}}\left(q^{|\eta|}\right) \cap G, \quad r \in \Phi^{p r}, \quad 1 \leqq i \leqq N
$$

with elements $x_{r, i}^{\mathrm{sub}}(t)$ or $x_{r, i}^{\mathrm{sub}}(t, u)$. The parametrization is as follows.

$r$ a long root: $r \in \Phi^{P r} \cap \Phi, x_{r, i}^{\mathrm{sub}}(t)=x_{r}(t), t=t^{q}$ if $\eta \neq \nu$ and $t=$ $-t^{q}$ if $\eta=\nu$. (See the proof of (3.12).)

$r$ a short root, $\eta \neq \psi: \quad x_{r, i}^{\text {sub }}(t)=x_{s}(t) \cdot x_{\eta(s)}\left( \pm t^{q}\right)$ with $s, \eta(s) \in \Phi_{\imath}^{\text {sub }} \cap$ $\operatorname{Pr}^{-1}(r)$.

$r$ a half-root, $\eta=\nu: x_{r, i}^{\mathrm{sub}}(t, u)=x_{s}(t) \cdot x_{\nu(s)}\left( \pm t^{q}\right) \cdot x_{s+\nu}(s)(u)$ with $u+$ $u^{q}= \pm t t^{q}$. (See the proof of (3.12).)

$r$ a short root, $\eta=\psi: \quad x_{r, i}^{\mathrm{sub}}(t)=x_{s}(t) \cdot x_{\psi(s)}\left( \pm t^{q}\right) \cdot x_{\psi \psi^{2}(s)}\left( \pm t^{q^{2}}\right)$ with $s, \psi(s), \psi^{2}(s) \in \Phi_{i}^{\text {sub }} \cap \operatorname{Pr}^{-1}(r)$.

If $r$ is a long root in $\Phi^{p r}$, the groups $\mathscr{Z}_{r, i}^{\text {sub }}$ coincide for $i=1$, $\cdots, N$. If not, there are $|\eta|$ different groups for $i=1, \cdots, N$.

Between the elements of $\mathscr{X}_{r, i}^{\text {sub }}$ and $\mathscr{X}_{s, i}^{\text {sub }}, r, s \in \Phi^{P r}$, there are commutator relations as described in Steinberg [4, p. 181]. In the case $\eta=\psi$ there is another nontrivial commutator relation of the type $\left[\mathscr{X}_{r, i}^{\mathrm{sub}}, \mathscr{X}_{s, j}^{\mathrm{sub}}\right] \subseteq \mathscr{X}_{r+s, \mathrm{k}}^{\mathrm{sub}}$ where $r, s, r+s$ are short roots in $\Phi^{P r}=G_{2}$ and $i \neq j \neq k \neq i$.

(5.6) Lemma. Let $\Omega$ be a component of $\Phi^{\mathrm{ker}}$. The elements in $\Omega\left(q^{|\eta|}\right) \cap G$ which for every $r \in \Phi^{P r}$ permute the rootgroups $\mathscr{Z}_{r, i}^{\mathrm{sub}}, i=1$, $\cdots, N$ are, with notation from (3.3) and (3.5):

$$
\begin{gathered}
\left\{\gamma\left(\begin{array}{cc}
v^{q} & 0 \\
0 & v
\end{array}\right)\right\} \cup\left\{\gamma\left(\begin{array}{cc}
0 & u^{q} \\
u & 0
\end{array}\right)\right\} \quad \text { if } \eta \neq \psi \text {, } \text { and } \\
\left\{\gamma\left(\begin{array}{ccc}
z^{q^{2}} & 0 & 0 \\
0 & z^{q} & 0 \\
0 & 0 & z
\end{array}\right)\right\} \cup\left\{\gamma\left(\begin{array}{ccc}
0 & a c x^{q^{2}} & 0 \\
0 & 0 & b c x^{q} \\
x & 0 & 0
\end{array}\right)\right\} \cup\left\{\gamma\left(\begin{array}{ccc}
0 & 0 & b c y^{q^{2}} \\
a b y^{q} & 0 & 0 \\
0 & y & 0
\end{array}\right)\right\} \text { if } \eta=\psi
\end{gathered}
$$

Proof. This is checked by means of matrix models of the $K 4 K\left(q^{2}\right)$ groups and $K K 9 K K\left(q^{3}\right)$-groups as indicated in (3.8) or the model of the $5 K 5\left(q^{2}\right)$-groups in (3.10). Note that these elements are $\eta$-invariant 
elements of the monomial group of $\Phi\left(q^{|\eta|}\right)$.

(5.7) Let $U_{i}^{\text {sub }}, 1 \leqq i \leqq N$, be the $p$-Sylowgroup $(p=\operatorname{char} G F(q))$ of the twisted group ${ }^{|\eta|} \Phi_{i}^{\text {sub }}\left(q^{|n|}\right)$ defined by $U_{i}^{\text {sub }}=U \cap \Phi_{i}^{\text {sub }}\left(q^{|n|}\right) \cap G$ with $U$ as in (4.11), i.e.,

$$
U_{i}^{\mathrm{sub}}=\prod_{r \in \mathscr{P}^{P+}+} \mathscr{Z}_{r, i}^{\mathrm{sub}} .
$$

$$
\text { Let } U^{P r+}=\left\langle U_{i}^{\mathrm{sub}} ; 1 \leqq i \leqq N\right\rangle=\prod_{r \in \phi^{P} r+}\left(\mathscr{Y}_{r} \cap G\right) \text {. }
$$

Expressing $\mathscr{Y}_{r} \cap G$ in each of the two descriptions of $G$ we get because of (5.1)

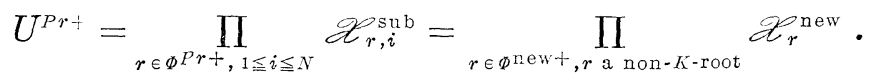

(5.10) Lemma. Let $H$ be the highest root in $\Phi^{P r+}$. Then $H \in \Phi^{P r} \cap \Phi \cap$ $\Phi^{\text {new }}$ and $\mathscr{Z}_{H} \cap G=\mathscr{X}_{H}^{\text {new }}=Z\left(U^{P r+}\right)=\mathscr{X}_{H, i}^{\text {sub }}=Z\left(U_{i}^{\text {sub }}\right), 1 \leqq i \leqq N$.

Proof. The highest root in $\mathscr{\Phi}^{\text {Pr+ }}$ is long, hence $H$ is an $L$-root in $\Phi$, and $H \in \Phi^{P r} \cap \Phi \cap \Phi^{\text {now }}$. The rest is easily checked with commutator formulas in $G$ using the first description above and in the twisted subgroups using the second description.

(5.11) Let $R$ be the set of fundamental roots in (5.5). Let $J \subset R$. We recall that the parabolic subgroup $P_{J}$ defined by $J$ has a normal series

$$
P_{J} \triangleright P_{J}^{*} \triangleright O_{p}\left(P_{J}\right), \quad p=\operatorname{char} G F(q)
$$

where $P_{J}^{*}$ is generated by $O_{p}\left(P_{J}\right)$ and the rootgroups $\mathscr{X}_{r}^{\text {new }}$ with $r$ or $-r$ expressible by the roots in $J . P_{. J}$ is generated by $P_{J}^{*}$ and the diagonal subgroup of $G$, so the index $\left[P_{J}: P_{J}^{*}\right]$ is prime to $p$. $\quad P_{J}^{*} / O_{p}\left(P_{J}\right)$ is isomorphic to the direct product of the Chevalley groups (twisted or not) defined by the connected components of $J$ (regarded as a subset of the Dynkin diagram).

(5.12) The maximal parabolic subgroup $P_{R-\{r\}}$ will be denoted simpler by $P_{R-r}$ and the parabolic subgroup defined by all $K$-roots in (5.5) will be denoted $P_{K}$.

The main result of this section is

(5.13) THeorem. $\quad N_{G}\left(U_{0}^{\text {sub }}\right) \subset P_{K}, \quad 1 \leqq i \leqq N$.

Proof. Let $B$ be as in (5.5) and $H$ as in (5.10). Then $(B, H)>0$ and $(r, H)=0$ for $r \neq B, r$ fundamental in (5.5). Hence, using (5.10), 


$$
N_{G}\left(U_{i}^{\text {sub }}\right) \cong N_{G}\left(Z\left(U_{i}^{\text {sub }}\right)\right)=N_{G}\left(\mathscr{Z}_{H}^{\text {new }}\right)=P_{R-B} .
$$

We want to show that $N_{G}\left(U_{i}^{\text {sub }}\right) \cong P_{R-A}$ with $A$ as in (5.5). This is clear if $\eta \neq \lambda$, because then $A=B$.

(5.14) If $\eta=\lambda$, we argue as follows: Suppose $y \in N_{G}\left(U_{i}^{\text {sub }}\right)$, and $y=u^{\prime} h n_{w} u$ in the Bruhat decomposition of $G$ associated with the first description above. Since $y \in P_{R-B}, w=w_{A}^{a} \cdot w^{\prime}$ where $w_{A}$ is the reflection along $A$ and $w^{\prime}$ is expressible by reflections along fundamental roots difierent from $A$ and $B$. Clearly $a=0$ or $a=1$, and we will show that $a=0$. Since $A$ is an $L$-root, $\mathscr{X}_{A} \cap G=\mathscr{X}_{A, i}^{\text {sub }}=$ $\mathscr{Z}_{A}^{\text {new }} \subset U_{i}^{\text {sub }} \subset U^{P r+}$. Let $1 \neq x_{A}^{\text {new }}(t)$. Then, using commutator formulas, $u \cdot x_{A}^{\text {new }}(t) \cdot u^{-1}=x_{A}^{\text {new }}(t) \cdot \prod_{s>A} x_{s}^{\text {new }}\left(t_{s}\right)$ for certain $t_{s}$. Hence if $a=1$, $n_{w} u \cdot x_{A}^{\text {new }}(t) \cdot u^{-1} n_{w}^{-1} \notin U^{P r+}$ and consequently $y \cdot x_{A}^{\text {new }}(t) \cdot y^{-1} \notin U^{P r+}$. This implies $y \notin N_{G}\left(U_{i}^{\text {sub }}\right)$. Hence $a=0$ and $y \in P_{R-A}$.

It is now enough to compute modulo $O_{p}\left(P_{R-A}\right)$. The non- $K$ fundamental roots in (5.5) are removed one by one. The detached $K$ 's define direct factors as described in (5.11), so the situation repeats itself.

If $\eta=\psi$, the 9-root in (5.5) can be removed by an easy calculation with $6 \times 6$-matrices. If $\eta \neq \psi$, the non- $K$ fundamental roots are removed by repeating the argument, starting with $C$. If $\eta \neq \nu$, the last two steps are removal of a 4-root and an $L$-root as in the argument (5.14). If $\eta=\nu$, the last step is removal of the short root in the subsystem $K \circ=0$, an easy verification.

$$
\text { Corollary. Let } y \in N_{G}\left(U_{i}^{\text {sub }}\right) \text {. Then }
$$

$$
y=h \cdot\left(\prod_{\Omega \subset \emptyset \mathrm{kor}} y_{\Omega}\right) \cdot y^{+}
$$

with $h$ in the diagonal group of $G, y_{\Omega} \in \Omega\left(q^{|x|}\right) \cap G, y^{+} \in U^{P r+}$ and $y_{\Omega}$ as in Lemma (5.6).

Proof. The decomposition of $y$ follows from Theorem (5.13) and the description (5.11) of parabolic groups. Note that $O_{p}\left(P_{K}\right)=U^{P r+}$. Because of (4.6) $y_{\Omega}$ normalizes $\mathscr{Y}_{r} \cap G$ for all $r \in \Phi^{P r}$. It is checked with the models of (3.8) and (3.10) that $y_{\Omega}$ is as in (5.6).

(5.16) Let $E$ be a finite group, $D$ a subgroup and $p$ a prime. We will say that $D$ is $p$-maximal in $E$ if for a subgroup $F$ with greater $p$-part than $D, D \subset F \cong E$ implies $F=E$.

(5.17) Corollary. If $\eta \neq \psi$ and $p \neq 2$ or if $\eta=\nu$, then ${ }^{|\eta|} \Phi_{i}^{\text {sub }}\left(q^{|\eta|}\right)$ is p-maximal in $G, p=\operatorname{char} G F(q)$. 
Proof in outline. If not, we may suppose that $y \in N_{G}\left(U_{i}^{\text {sub }}\right)-U_{i}^{\text {sub }}$ is a $p$-element. By (5.15) and the argument of (1.11), $y \in U^{P r+}$. Then we may assume that

$$
1 \neq y=\prod_{r \in \mathscr{P}^{P}+} x_{r, j(r)}^{\mathrm{sub}}\left(t_{r}\right) \quad \text { with } \mathscr{\mathscr { X }}_{r, j(r)}^{\mathrm{sub}} \not \subset U_{i}^{\mathrm{sub}} .
$$

Taking commutators between $y$ and elements from $U_{i}^{\text {sub }}$ we may reduce to the case with just one term in the product. Conjugation by elements from the monomial group of $|\eta| \Phi_{i}^{\text {sub }}\left(q^{|\eta|}\right)$ then yields sufficiently many elements to show that $\left\langle y\right.$, $\left.{ }^{|\eta|} \Phi_{i}^{\text {sub }}\left(q^{|\eta|}\right)\right\rangle=G$.

In particular this shows that if $p \neq 2$ the embedding ${ }^{2} D_{m+1}\left(q^{2}\right) \subset$ $D_{m+2}(q)$ obtained for $\eta=\lambda$, cannot be refined to the sequence

$$
{ }^{2} D_{m+1}\left(q^{2}\right) \subset B_{m+1}(q) \subset D_{m+2}(q)
$$

which may be obtained in orthogonal geometry by choosing appropriate nonisotropic vectors and their orthogonal complements. See e.g. Artin [1, p. 147].

\section{REFERENCES}

1. Artin, Geometric Algebra, Intersc. Publ., N. Y., 1957.

2. Bourbaki, Groupes et algebres de Lie, Ch. IV, V, VI, Hermann, Paris, 1968.

3. Carter, Simple Groups of Lie Type, John Wiley \& Sons, 1972.

4. Steinberg, Lectures on Chevalley Groups, Yale Univ., 1967.

Received July 3, 1973.

The Norwegian School of Economics and Business Administration 